\title{
Influência da participação de estudantes em ligas acadêmicas na escolha da especialidade para o Programa de Residência Médica da Bahia 2017
}

\author{
Influence of student participation in academic leagues in the choice of specialty \\ for the Bahia 2017 Medical Residency Program
}

\author{
Sara de Moura Pontes ${ }^{1}$, Lara de Araújo Torreão ${ }^{2}$
}

Pontes SM, Torreão LA. Influência da participação de estudantes em ligas acadêmicas na escolha da especialidade para o programa de residência médica da Bahia 2017 / Influence of student participation in academic leagues in the choice of specialty for the Bahia 2017 Medical Residency Program. Rev Med (São Paulo). 2019 maio-jun.;98(2):160-7.

RESUMO: Introdução: A participação em Ligas Acadêmicas (LAs), hoje, é um importante componente do processo formativo do estudante de Medicina. No entanto, esta participação pode estar propiciando precocemente a escolha da especialidade futura, limitando a atuação e a busca por conhecimento amplo e generalizado. Objetivo: Avaliar a influência da participação em LAs sobre a escolha da especialidade por recém-ingressos no Programa de Residência Médica da Bahia 2017 (PRM/BA/2017). Métodos: Estudo epidemiológico observacional transversal, no qual residentes do PRM/BA/2017, durante matrícula, foram convidados a responder questionário contendo informações sobre a participação em LAs e a escolha da especialização médica. Resultados: Dos 339 entrevistados, 79,8\% participaram de LAs. Destes, 45,4\% consideraram que a participação em LAs influenciou a escolha da especialidade. A correlação geral entre participação em LAs e escolha de especialidade na mesma área (correlação L-E) atingiu 30,9\% e foi encontrada aumentada de maneira significativa no grupo que considerou que a participação em LAs influenciou o processo decisório, que participou da LA durante mais de 3 semestres, que ocupou 3 ou 4 cargos em uma mesma liga, que participou de "Estágios" e que alegou "Afinidade/ Curiosidade" como principal motivo para entrada na LA. Das grandes áreas da Medicina, o grupo que optou por Cirurgia Geral apresentou maior correlação L-E $(53,1 \%)$ e o que optou pela Pediatria menor (12,5\%) Conclusões: Apesar de estar presente no processo formativo da maioria dos estudantes, a participação em LAs não pareceu representar a especialização precoce destes. $\mathrm{O}$ fator mais associado à correlação L-E foi a percepção de que esta participação influenciou a escolha da especialidade.

Descritores: Internato e residência; Educação médica; Educação superior; Estudantes de medicina.

\begin{abstract}
Introduction: Participating in Academic Leagues (LAs) nowadays is an important component of the medical student's graduation process. However, this participation may be prematurely promoting specialty choice, limiting the performance and the search for ample and widespread knowledge. Objective: Evaluate the influence of participating in LAs with regards to the choice of specialty by new entrants in the Medical Residency Program of Bahia 2017 (PRM/BA/2017). Methodology: This is an observational cross-sectional epidemiological study in which residents from the PRM/BA/2017, during the enrollment, were invited to respond a questionnaire containing information about the participation in LAs and the choice of medical specialization. Results: 339 individuals were interviewed. $79.8 \%$ of interviewed participated in LAs. From those, $45.4 \%$ considered that participating in LAs influenced the choice of specialty. The general correlation between participation in LAs and the choice of a specialty at the same area (L-E correlation) reached $30.9 \%$. A higher and significant L-E correlation was found in group that considered participation in LA as a influence in the decision making process, that participated in LA for more than 3 semesters, that occupied 3 or 4 positions at the same league, that participated on "Internship" and that claimed "Affinity/ Curiosity" as the main reason for get into LA. Within all the medical specialties, the group that have chosen General Surgery had higher L-E correlation and the one that chose Pediatrics had lower. Conclusions: Despite being present in the training process of most students, participating in LAs did not seem to represent their premature specialization. The most associated factor with the L-E correlation was the perception that this participation influenced the choice of the specialization.
\end{abstract}

Keywords: Internship and residence; Education, medical; Education, higher; Students, medical.

1. Acadêmica da Faculdade de Medicina da Universidade Federal da Bahia - FMB/UFBA. ORCID: https://orcid.org/0000-0002-72582519. E-mail: sara_mpontes@yahoo.com.br.

2. Professora Adjunta II do Departamento de Pediatria da Faculdade de Medicina da Universidade Federal da Bahia. ORCID: https:// orcid.org/0000-0002-6328-2864. E-mail: lara.torreao@gmail.com.

Endereço para correspondência: Sara de Moura Pontes. Rua Deputado Eliel Martins, 7. Stella Maris. Salvador, Bahia, Brasil. CEP: 41600-630. 


\section{INTRODUÇÃO}

\begin{abstract}
A s Ligas Acadêmicas (LAs) podem ser caracterizadas como organizações estudantis sem fins lucrativos que criam para seus membros oportunidades de atividades didáticas, científicas, culturais e sociais, abrangendo sempre uma determinada área da saúde, visando seu aprendizado e desenvolvimento, sendo gerida pelos próprios estudantes, mas com orientação de docentes ${ }^{1}$.
\end{abstract}

Segundo, Rego² (p.10), "currículo paralelo" é o "conjunto de atividades extracurriculares que os alunos desenvolvem, subvertendo, na maioria das vezes, a estrutura curricular formal estabelecida pela Faculdade".

Como exemplo da importância que as LAs exercem no "currículo paralelo"2 do estudante de Medicina, Peres et al. ${ }^{3}$ mostraram que a participação em LAs foi a atividade mais frequentemente relatada pelos estudantes do $1^{\circ}-3^{\circ}$ ano, e "aproximar-se da prática médica" foi o principal motivo apontado nesse quesito.

Detalhando-se o funcionamento e a organização interna das LAs, existem cargos hierarquizados e diretorias de atividades centrais necessárias ao funcionamento desta organização, como: presidência, vice-presidência, secretaria, tesouraria, diretorias de ensino, pesquisa, extensão, comunicação. Desta maneira, pode-se dizer que as LAs ainda funcionam como espaço de desenvolvimento de competências de gestão e liderança, pouco exercidas nos currículos tradicionais.

Ferreira et al. ${ }^{4}$ ao tentarem fazer um paralelo entre LAs e "Learning Communities", grupo de aprendizado encontrado nas Universidades canadenses e americanas, frisam especificidades vistas nas LAs brasileiras que as tornam únicas como atividade extracurricular, são elas: autonomia do estudante na condução das atividades, organização baseada no tripé universitário (pesquisa/ ensino/extensão), variabilidade de participantes sob o aspecto de período no curso e origem de distintas Faculdades e inserção destas organizações em congressos, conferências, eventos regionais e nacionais da área em questão.

Sabendo de todas as funções e contribuições que as LAs desempenham no currículo e formação médicos, existem críticas e ponderações a serem feitas acerca do fenômeno frequente de participação nas mesmas. PêgoFernandes et al. ${ }^{1}$ apontam como aspecto negativo das LAs o fato de alguns alunos tomarem essas atividades como uma chance para "especialização precoce", dedicandose excessivamente a alguma área e relegando a segundo plano outras também importantes para formação médica generalista.

Um fato que corrobora este fenômeno foi observado por Monteiro et al. ${ }^{5}$, onde ao analisarem a participação de 14 estudantes na Liga Baiana de Cirurgia Plástica (LBCP) da Faculdade de Medicina da Bahia da Universidade Federal da Bahia nos anos de 2006 e 2007, a taxa de alunos que se interessava em atuar futuramente na área era de $28,6 \%$ antes da participação de liga e evoluiu para 78,6\% em um ano de participação.

Desta maneira, as LAs propiciariam a escolha do rumo profissional de maneira precoce, limitando a atuação e a busca por conhecimento amplo e generalizado, e, principalmente, indo de encontro às diretrizes curriculares nacionais (DCN) para a formação médica consonante com as demandas atuais. Este fato levanta a necessidade de uma análise do fenômeno, a fim de possibilitar o desenvolvimento de estratégias dentro do modelo de tais organizações para reduzir uma possível restrição.

Assim, este trabalho visa investigar a correlação entre a participação em LAs de estudantes do Programa de Residência Médica da Bahia e a escolha da especialidade médica no ano de 2017. Objetiva-se desta forma explorar a associação entre atuação nas Ligas em termos de tempo, cargos ocupados e atividades desenvolvidas nestas e a escolha pelo programa de residência médica de área correlata, delineando melhor a função das LAs no processo formativo médico.

\section{MATERIAIS E MÉTODOS}

Estudo epidemiológico transversal aprovado pelo Comitê de Ética em Pesquisa em Seres Humanos da Faculdade de Medicina da Universidade Federal da Bahia (CAAE 62911816.0.0000.5577). Os dados foram coletados através de aplicação presencial de questionários, após consentimento (Termo de Consentimento Livre e Esclarecido - TCLE), no momento da matrícula dos residentes no Programa de Residência Médica Bahia 2017 (PRM/BA 2017), que ocorreu em Salvador-BA no período de 13/02/2017 a 17/02/2017. O questionário aplicado continha perguntas em formato de alternativas e semidissertativas acerca de dados demográficos (gênero, idade, Faculdade de Medicina onde se graduou, tempo de formado), participação em ligas acadêmicas (natureza da liga, tempo de participação, cargos ocupados, atividades desenvolvidas, motivação principal para a entrada na liga, influência sobre a escolha da especialidade médica) e a opção de especialidade de acesso direto ou com prérequisito no PRM/BA 2017. Os questionários preenchidos de maneira ilegível, parcial ou inadequada foram excluídos.

Para analisar a correlação entre a participação em LAs e a escolha da especialidade médica, foi definido que correlação liga - especialidade (correlação L-E) estava presente nas seguintes situações:

1) Entrevistado participou da Liga da própria especialidade escolhida. Ex.: Participou da Liga de Pediatria e especialidade a ser matriculada Pediatria;

2) Entrevistado participou de Liga de especialidade que necessita como pré-requisito para entrada a especialidade que será feita neste momento. Ex.: 
Participou de Liga de Cardiologia e especialidade a ser matriculada Clínica Médica;

3) Entrevistado participou de Liga de especialidade que é pré-requisito para a especialidade que será matriculada agora. Ex.: Participou de Liga de Clínica Médica e especialidade a ser matriculada Cardiologia;

4) Entrevistado participou de Liga de área de atuação que necessita como pré-requisito para sua entrada a especialidade que será feita neste momento. Ex.: Participou de Liga de Vídeocirurgia e especialidade a ser matriculada Cirurgia Geral;

5) Entrevistado participou de Ligas com nomes altamente sugestivos de correlação com especialidades ou áreas de atuação conhecidas. Ex.: Participou de Liga de DST/AIDS, que está correlacionada à especialidade de Infectologia, Liga de Saúde da Mulher está correlacionada à Ginecologia e Obstetrícia etc.;

6) Entrevistado participou de Ligas de especialidades intimamente ligadas a outras. Ex.: Liga de Oncologia está para as especialidades ou área de atuação Hematologia, Cancerologia Clínica, Cancerologia Cirúrgica, Cancerologia Pediátrica ou Radioterapia; Liga de Anatomia Clínico-Cirúrgica ou Anatomia Cirúrgica está para Cirurgia Geral; Liga de Medicina Legal está para Patologia.

Foram consideradas correlação "indeterminada" as participações em ligas de áreas transversais como: Emergência, Trauma, Anatomia, Anatomia Clínica, Farmacologia, Fisiologia, Fisiopatologia, Semiologia Médica, Medicina Interna, Nutrição Esportiva, Dor, Saúde e Espiritualidade etc.

Os dados foram tabulados no programa Excel 2013 (Microsoft) e analisados usando o programa GraphPad Prism 7® (GraphPad Software Inc.). As variáveis contínuas de distribuição normal foram descritas em valores médios, medianas, modas e desvios-padrão. As variáveis categóricas foram descritas em proporções. Foram realizadas comparações entre as variáveis categóricas através do teste qui-quadrado para os dados com valores numéricos $>15$ e através do teste exato de Fisher para os dados com valores numéricos $<$ ou iguais a 15 . O intervalo de confiança (IC) foi de $95 \%$ com erro alfa aceito de 5\%.

\section{RESULTADOS}

Das 692 vagas oferecidas pelos PRM/BA 2017 entre especialidades de acesso direto e com pré-requisito, conseguimos entrevistar 346 indivíduos, sendo que destes, 7 questionários foram preenchidos parcialmente ou de maneira inadequada ou ilegivelmente e 2 não constavam assinatura do TCLE. Assim, foram incluídos no estudo 337 questionários.

A Tabela 1 mostra as características sociodemográficas dos entrevistados.
Tabela 1. Características sociodemográficas de 337 recémingressos no Programa de Residência Médica da Bahia 2017

\begin{tabular}{|c|c|}
\hline Característica & Valor - n (\%) \\
\hline \multicolumn{2}{|l|}{ Sexo } \\
\hline Feminino & $202(59,9 \%)$ \\
\hline Masculino & $135(40,1 \%)$ \\
\hline \multicolumn{2}{|l|}{ Idade } \\
\hline $21-25$ anos & $91(27 \%)$ \\
\hline 26-30 anos & $195(57,9 \%)$ \\
\hline $31-35$ anos & $39(11,6 \%)$ \\
\hline $36-40$ anos & $9(2,7 \%)$ \\
\hline 41 anos ou mais & $3(0,9 \%)$ \\
\hline \multicolumn{2}{|c|}{ Faculdades: Nacionalidade } \\
\hline Brasileira & $330(97,9 \%)$ \\
\hline Estrangeira & $5(1,5 \%)$ \\
\hline Não especificadas & $2(0,6 \%)$ \\
\hline \multicolumn{2}{|c|}{ Faculdades Brasileiras: Natureza } \\
\hline Pública & $130(39,4 \%)$ \\
\hline Privada & $200(60,6 \%)$ \\
\hline \multicolumn{2}{|c|}{ Faculdades Brasileiras: Regiões } \\
\hline Nordeste & $275(83,3 \%)$ \\
\hline Sudeste & $39(11,8 \%)$ \\
\hline Norte & $10(3 \%)$ \\
\hline Sul & $5(1,5 \%)$ \\
\hline Centro-Oeste & $1(0,3 \%)$ \\
\hline \multicolumn{2}{|c|}{ Faculdades Nordestinas: Estados } \\
\hline Bahia & $215(78,2 \%)$ \\
\hline Paraíba & $17(6,2 \%)$ \\
\hline Ceará & $16(5,8 \%)$ \\
\hline Sergipe & $13(4,7 \%)$ \\
\hline Pernambuco & $9(3,3 \%)$ \\
\hline Maranhão & $3(1,1 \%)$ \\
\hline Piauí & $2(0,7 \%)$ \\
\hline \multicolumn{2}{|c|}{ Faculdades Baianas: Instituições } \\
\hline BAHIANA & $97(45,1 \%)$ \\
\hline UFBA & $48(22,3 \%)$ \\
\hline FTC & $29(13,5 \%)$ \\
\hline UESC & $19(8,8 \%)$ \\
\hline UESB & $13(6,0 \%)$ \\
\hline UEFS & $9(4,2 \%)$ \\
\hline \multicolumn{2}{|l|}{ Tempo de graduado } \\
\hline $0-2$ anos & $204(60,5 \%)$ \\
\hline 2 anos e 1 dia -4 anos & $96(28,5 \%)$ \\
\hline 4 anos e 1 dia -6 anos & $25(7,4 \%)$ \\
\hline 6 anos e 1 dia ou mais & $12(3,6 \%)$ \\
\hline
\end{tabular}


Pontes SM, Torreão LA. Influência da participação de estudantes em ligas acadêmicas.

Quanto à participação em LAs durante a graduação, dos 337 entrevistados, 269 indivíduos (79,8\%) disseram ter participado desta atividade, sendo que destes, $68,4 \%$ ingressaram em uma única liga durante toda graduação e o restante participou de mais de uma Liga Acadêmica. Assim contabilizamos 371 respostas acerca de participação em LAs.
A Tabela 2 sumariza a descrição das 371 participações em LAs relatadas pelos 269 estudantes entrevistados que fizeram parte de alguma liga durante a graduação, quanto a quantidade, tempo de participação, influência, cargos ocupados, atividades desenvolvidas e motivações para entrada na liga.

Tabela 2. Descrição da participação em Ligas Acadêmicas (LA) durante a graduação de 269 recém-ingressos no Programa de Residência Médica da Bahia 2017

\begin{tabular}{|c|c|}
\hline \multicolumn{2}{|l|}{ Quantidade } \\
\hline 1 Liga & $184(68,4 \%)$ \\
\hline 2 Ligas & $68(25,3 \%)$ \\
\hline 3 Ligas & $17(6,3 \%)$ \\
\hline Tempo de participação & Em semestres \\
\hline Média & 3 \\
\hline Mediana & 2 \\
\hline Influência da participação em LA sobre a escolha da especialidade & Valor - n (\%) \\
\hline Sim & $122(45,4 \%)$ \\
\hline Não & $140(52 \%)$ \\
\hline Indeterminado & $7(2,6 \%)$ \\
\hline \multicolumn{2}{|l|}{ Cargos ocupados na LA (Total: Relatos de 328 cargos ocupados) } \\
\hline Indeterminado & $105(28,3 \%)$ \\
\hline \multicolumn{2}{|l|}{ Quantidade de cargos ocupados em uma mesma liga } \\
\hline 1 & $222(83,5 \%)$ \\
\hline 2 & $28(10,5 \%)$ \\
\hline 3 & $14(5,3 \%)$ \\
\hline 4 & $2(0,7 \%)$ \\
\hline \multicolumn{2}{|l|}{ Relatos de ocupação de cargos } \\
\hline Presidência & $42(12,8 \%)$ \\
\hline Vice-presidência & $41(12,5 \%)$ \\
\hline Tesouraria & $34(10,4 \%)$ \\
\hline Secretaria & $54(16,5 \%)$ \\
\hline Diretorias (ensino, pesquisa, extensão, comunicação) & $167(47,9 \%)$ \\
\hline \multicolumn{2}{|l|}{ Atividades desenvolvidas na LA (Total: Relatos de 1072 atividades desenvolvidas) } \\
\hline Indeterminado & $11(3 \%)$ \\
\hline \multicolumn{2}{|l|}{ Quantidade de atividades desenvolvidas em uma mesma liga } \\
\hline 1 & $66(1,3 \%)$ \\
\hline 2 & $79(21,9 \%)$ \\
\hline 3 & $77(21,4 \%)$ \\
\hline 4 & $73(20,3 \%)$ \\
\hline 5 & $65(18,1 \%)$ \\
\hline \multicolumn{2}{|l|}{ Relatos de atividades desenvolvidas } \\
\hline Eventos & $282(26,3 \%)$ \\
\hline Estágios & $245(22,9 \%)$ \\
\hline Ensino & $242(22,6 \%)$ \\
\hline Extensão & $157(14,6 \%)$ \\
\hline Pesquisa & $146(13,6 \%)$ \\
\hline \multicolumn{2}{|l|}{ Motivação para participar da LA (Total: 416 motivações relatadas) } \\
\hline Indeterminado & $17(4,6 \%)$ \\
\hline Afinidade/Curiosidade & $292(70,2 \%)$ \\
\hline Aprender algo específico/Dificuldade na área & $105(25,2 \%)$ \\
\hline $\begin{array}{l}\text { Pertencimento - "todos os colegas faziam parte de alguma liga à época, assim me senti induzido a participar } \\
\text { desta" }\end{array}$ & $16(3,8 \%)$ \\
\hline Outros & $3(0,7 \%)$ \\
\hline
\end{tabular}


Em relação à correlação L-E, isto é, participação em Liga de determinada área e escolha por especialidade em área correlata, já detalhada previamente, demonstrase que esta correlação foi positiva em 30,9\% dos 269 respondedores. O Gráfico 1 mostra as porcentagens de correlação L-E encontradas.

Analisando a correlação L-E separadamente quanto às especialidades que representam as grandes áreas da Medicina (Tabela 3), nota-se que na "Cirurgia Geral”, um pouco mais da metade dos estudantes (53,1\%) participaram de Ligas em área correlata. Para a "Clínica Médica" e "Ginecologia e Obstetrícia", a correlação alcançada assemelhou-se ao encontrado para a amostra geral, $36,2 \%$ e $29,4 \%$, respectivamente. $\mathrm{Na}$ "Pediatria", obteve-se percentagem muito pequena de correlação L-E (12,5\%). E para "Medicina de Família e Comunidade", dado o pequeno número de entrevistados, não obtivemos qualquer nível de correlação.

Comparamos grupos separados por especificidades de participação e a Tabela 4 mostra os valores de correlação L-E encontrados em cada grupo, bem como se esta especificidade esteve associada significativamente com diferença de correlação L-E (valor p).
Gráfico 1. Correlação liga-especialidade (L-E) entre as 371 participações em Ligas Acadêmicas (LA) relatadas por 269 recém-ingressos no Programa de Residência Médica da Bahia 2017

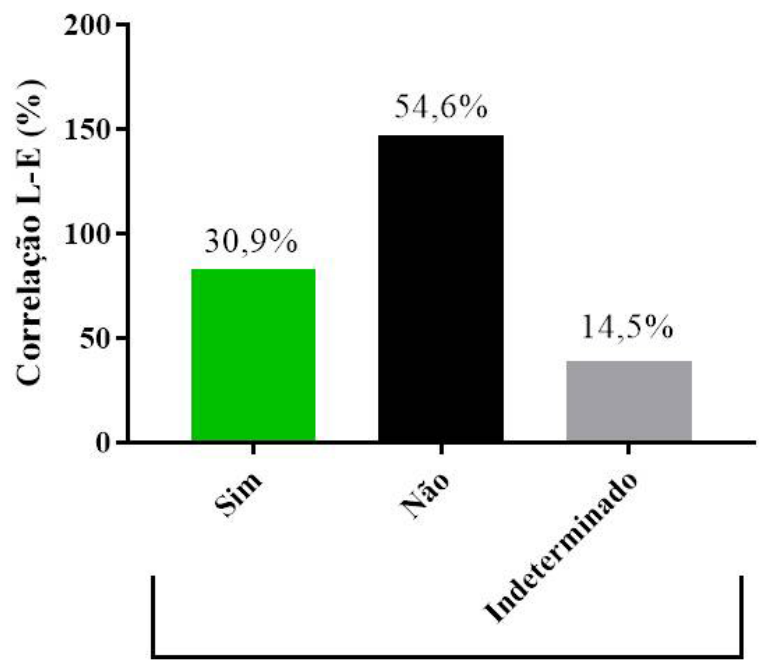

Participação em LA

Tabela 3. Participação em Ligas Acadêmicas e correlação com escolha de especialidade (correlação L-E) em estudantes selecionados para as grandes áreas no Programa de Residência Médica da Bahia no ano de 2017

\begin{tabular}{|c|c|c|c|c|}
\hline Grandes áreas & $\begin{array}{c}\text { Quantidade de vagas } \\
\text { oferecidas* }\end{array}$ & $\begin{array}{l}\text { Quantidade de questionários } \\
\text { respondidos n }(\%)\end{array}$ & $\begin{array}{l}\text { Participação em } \\
\text { Ligas n }(\%) \\
\end{array}$ & $\begin{array}{c}\text { Correlação L-E } \\
\text { n (\%) } \\
\end{array}$ \\
\hline Cirurgia Geral & 65 & $36(55,4 \%)$ & $32(88,9 \%)$ & $17(53,1 \%)$ \\
\hline Clínica Médica & 129 & $69(53,5 \%)$ & $58(85,3 \%)$ & $21(36,2 \%)$ \\
\hline Ginecologia e Obstetrícia & 35 & $19(54,3 \%)$ & $17(89,5 \%)$ & $5(29,4 \%)$ \\
\hline Medicina de Família e Comunidade & 52 & $4(7,7 \%)$ & $2(50 \%)$ & 0 \\
\hline Pediatria & 83 & $49(59 \%)$ & $32(65,3 \%)$ & $4(12,5 \%)$ \\
\hline
\end{tabular}

* De acordo Edital de Seleção do Programa de Residência Médica da Bahia 2017.

Tabela 4. Nível de correlação (\%) entre participação em Liga Acadêmica (LA) e especialidade escolhida (correlação L-E) em diferentes grupos separados por especificidades de participação entre recém-ingressos no Programa de Residência Médica da Bahia 2017

\begin{tabular}{|c|c|c|}
\hline Grupos & $\begin{array}{c}\text { Nível de correlação } \\
\text { L-E }(\%)\end{array}$ & Valor $\mathbf{p}$ \\
\hline Percepção de que a participação em LA influenciou a escolha da especialidade: (Total: 361 respostas) & & $<0,001$ \\
\hline Não & $26,5 \%$ & \\
\hline Sim & $81,3 \%$ & \\
\hline Tempo de participação da LA: (Total: 356 respostas) & & $<0,01$ \\
\hline Menor ou igual a 3 semestres & $25,7 \%$ & \\
\hline Maior que 3 semestres & $43,0 \%$ & \\
\hline Natureza do cargo ocupado na LA: (Total: 266 respostas) & & 0,389 \\
\hline Outros & $29,5 \%$ & \\
\hline Presidência/Vice-presidência & $36,4 \%$ & \\
\hline Quantidade de cargos ocupados em uma mesma LA: (Total: 266 respostas) & & $<0,05$ \\
\hline 1 ou 2 cargos & $29,5 \%$ & \\
\hline 3 ou 4 cargos & $57,1 \%$ & \\
\hline Natureza da atividade desenvolvida na LA: (Total: 360 respostas) & & $<0,05$ \\
\hline Outras & $22,8 \%$ & \\
\hline Estágios & $36,2 \%$ & \\
\hline Motivação para participar da LA: (Total: 341 respostas) & & $<0,01$ \\
\hline Curiosidade/Afinidade pela área & $35,9 \%$ & \\
\hline Aprender/Dificuldade na área & $6,9 \%$ & \\
\hline
\end{tabular}


Pontes SM, Torreão LA. Influência da participação de estudantes em ligas acadêmicas.

Após calcular as razões de chance (Odds-Ratio) de todos os fatores relacionados à participação na LA, que estiveram associados à correlação L-E de maneira significativa, obtivemos os resultados descritos no Gráfico 2.

Gráfico 2. Razão de chance de optar por especialidade em área correlata a da Liga Acadêmica (correlação L-E) a depender do fator em questão entre os recém-ingressos do Programa de Residência Médica da Bahia 2017

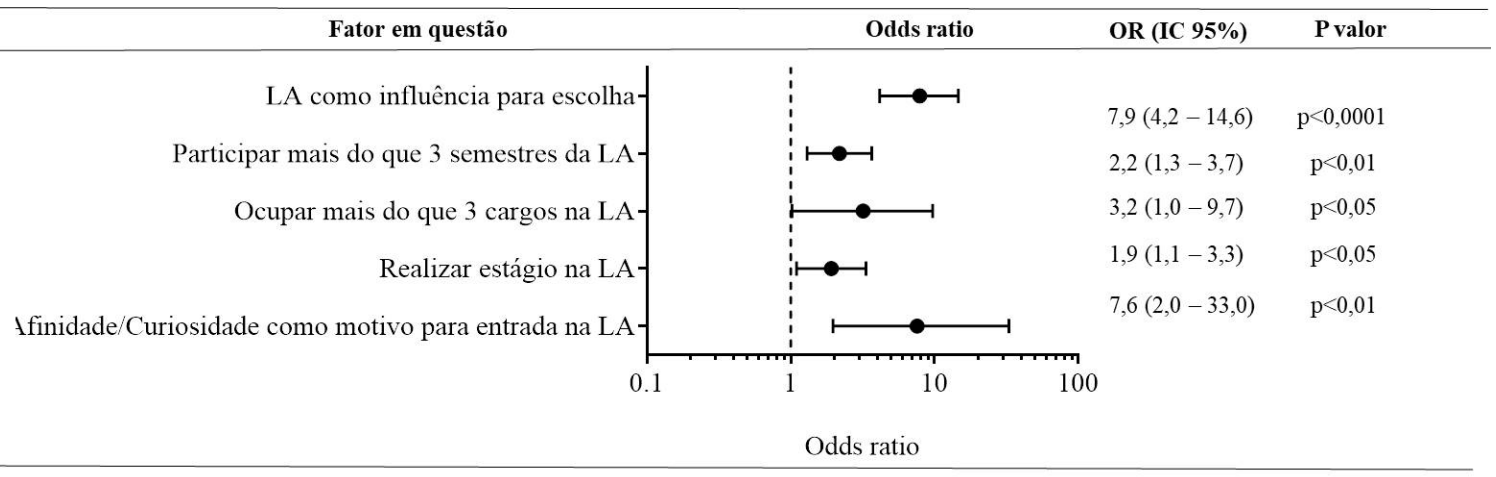

\section{DISCUSSÃO}

Em nosso estudo, quase $80 \%$ dos entrevistados participaram de Ligas Acadêmicas (LAs) durante a graduação, mostrando que as LAs figuram como atividade importante no currículo "paralelo" do graduando de Medicina.

Espaços estes, administrados pelos estudantes, sob orientação de um professor/profissional da área, nos quais competências como liderança, gestão, organização, promoção de eventos, ensino, entre outras, podem ser exercitadas. Assim, em uma realidade onde cada vez mais distintas habilidades são valorizadas, a participação em LAs se torna oportunidade frutífera de desenvolver capacidades outras que não só as técnicas-científicas, classicamente oferecidas no ambiente acadêmico. Além disso, a possibilidade de contato com a área de maneira mais intensa seja através de estágios prático-observacionais ou mesmo discussão de temas específicos motiva muitos estudantes a participarem desses espaços.

Buscou-se saber o nível de correlação LigaEspecialidade, isto é, o estudante ter participado de uma LA e em seguida optar por especialidade em área correlata. Foi encontrada correlação L-E de 30,9\% entre os 269 estudantes que participaram ao menos de uma LA durante a estadia na faculdade de Medicina (Gráfico 1). Esta porcentagem é menor do que a encontrada por Lima, ao entrevistar 133 ligantes de faculdades de Medicina da cidade de Belém, maioria cursando $1^{\circ}-3^{\circ}$ ano, os quais $44,11 \%$ afirmaram que o objeto de estudo da liga em que atuavam era a especialidade que pensavam em seguir ${ }^{6}$.

Devemos nos atentar ao fato de nesta amostra, os estudantes estarem no início do curso médico, diferente da nossa, composta por médicos, com decisão de especialidade já consumada. Existe ainda a possibilidade dos valores correlação L-E encontrados no nosso estudo serem maiores caso utilizássemos critérios de definição "correlação L-E” mais abrangentes - por exemplo, consideramos a área de Trauma multidisciplinar e a correlação não pôde ser determinada neste caso. Mas optamos por restringir esta definição, a fim de estabelecer um nível mais preciso e criterioso da influência da LA na escolha da especialidade.

Ao fazermos a análise de correlação L-E separadamente dentro das grandes áreas da Medicina (Tabela 3), notamos que "Cirurgia Geral" obteve maior nível de correlação L-E $(53,1 \%)$ do que a amostra geral, e a "Pediatria", menor (12,5\%). A "Clínica Médica" e a "Ginecologia e Obstetrícia" obtiveram valores de correlação próximos ao do geral com, respectivamente, $36,2 \%$ e $29,4 \%$.

O nível de correlação L-E encontrado para a Cirurgia Geral na nossa amostra foi maior que o mostrado por Simões 7 , no qual dos 363 egressos da Liga de Trauma da Universidade Estadual de Campinas (UNICAMP), 131 $(36,1 \%)$, optaram por fazer residência de Cirurgia Geral. $\mathrm{Na}$ amostra paulista, o autor considerou Trauma e Cirurgia Geral áreas correlatas, enquanto em nosso estudo, Trauma foi designada como área de correlação indeterminada, ou seja, o valor correlação L-E encontrado poderia ser ainda maior.

Diferentemente, Souza et al. ${ }^{8}$, em sua amostra de 1225 indivíduos entre médicos que prestariam concurso para residência médica no ano de 2013 (28,3\%) e estudantes de medicina dos dois últimos anos do curso $\left(9^{\circ}\right.$ a $12^{\circ}$ período) de faculdades públicas e particulares $(70,1 \%)$ do Rio de Janeiro e Salvador, mostraram que os estudantes que fizeram ligas de Pediatria, tiveram chance 9,5 vezes maior de referir a opção do que a população restante. Enquanto isso, em nosso estudo, obtivemos correlação menor que a geral. No mesmo sentido acima, Silva et al. ${ }^{9}$ mostraram que $64 \%$ dos 28 graduandos em Medicina da Universidade Gama Filho/RJ regularmente inscritos do $9^{\circ}$ ao $12^{\circ}$ período 
em 2012 que tinham a Pediatria como especialidade a ser seguida, participaram de LAs e monitorias na área. A correlação L-E encontrada para a Pediatria no nosso trabalho $(12,5 \%)$ foi muito baixa levando em conta a forte influência de participação em atividades extracurriculares descrita acima. Mais uma vez, a nossa amostra difere das citadas acima em relação ao fato de o processo de escolha já ter sido consumado, ao passo que entre graduandos existe apenas a intenção de escolha. Assim, pode ser que durante o processo de formatura e talvez ida para o mercado de trabalho existam fatores que possam estar alterando o fluxo decisório previamente estabelecido. Além disso, dentre as grandes áreas com número significante de estudantes respondedores no nosso estudo, a Pediatria foi a que apresentou menor porcentagem de estudantes que participaram de LAs (65,3\%, Tabela 3$)$.

Mesmo não obtendo correlação L-E geral significativa, decidimos analisar se havia associação entre diferentes fatores relativos à participação em LA com esta correlação. Ou seja, analisamos se alguns aspectos acerca da participação nessas organizações poderiam estar ligados a uma tendência de correlação L-E não observada inicialmente.

Em relação à percepção de que a participação em LA influenciou ou não a escolha da especialidade, foi mostrado que os estudantes que consideraram que a participação em LA foi importante para o momento decisório da especialidade tinham uma chance quase 8 vezes maior de apresentar correlação L-E (Gráfico 2), e este foi o fator isolado que pareceu estar mais associado à participação em Liga e escolha de especialidade nas mesmas áreas. Portanto, dentre que os que alegaram que a participação em LA influenciou o seu processo decisório, esta influência pareceu ser de natureza mais positiva e favorecedora, do que de rechaço ou distanciamento da área em questão (influência negativa).

Notamos que permanecer na LA mais do que 3 semestres aumentou a chance de escolha de especialidade na área em duas vezes. Possivelmente, maior tempo na LA aumenta a probabilidade de envolvimento e aprofundamento com a área em questão.

Quanto aos cargos ocupados na LA, embora exista uma organização hierarquizada de funcionamento, não notamos diferenças significativas entre os diferentes cargos ocupados e o nível de correlação L-E em cada grupo. Apenas os postos mais elevados hierarquicamente foram analisados ("Presidência" e "Vice-presidência"), já que nestas circunstâncias, poderia haver maior comprometimento com a LA, maior tempo de participação, maior proximidade com as atividades desenvolvidas etc. Mas estes fatores parecem ou não existir ou não serem importantes a ponto de trazer diferenças para os níveis de correlação L-E no futuro. A quantidade de cargos ocupados, por sua vez, esteve associada com maior chance de escolha da área correlata (Tabela 4 e Gráfico 2), mas estando relacionada com a variável "tempo de participação na LA”, e assim, não pudemos definir claramente a força de um ou outro fator em questão. Provavelmente, os dois fatos são relevantes, tendo o tempo como um fator que denota maior exposição, e a quantidade de cargos, como um sinal de maior compromisso, ambos propiciando maior correlação L-E.

Levando em conta a influência das experiências práticas sobre a escolha final, analisamos a correlação L-E sobre grupos que desenvolveram diferentes atividades na LA. Das atividades que elencamos em nosso questionário, a que mais se aproxima da rotina profissional diária foi "Estágios". Desta maneira, ao analisar o grupo que o fazia em relação a outro, que desempenhava outras atividades, aquele tinha quase duas vezes mais chances de escolher especialidade em área correlata à da LA (Gráfico 2). Nogueira-Martins et al. ${ }^{10}$ ao explorarem as percepções de estudantes do $5^{\circ}$ ano de Medicina da Universidade Federal de São Paulo mostraram grande anseio por parte destes pela vivência da prática clínica ainda no período pré-internato. Em nosso estudo, esta "aproximação da prática médica" 5 , por sua vez, , pareceu contribuir para a escolha de especialidade em área correlata a da vivenciada ainda na graduação. Provavelmente, esta aproximação foi positiva para os que a fizeram, reforçando a escolha pela área específica que já existia previamente ou criando no estudante a vontade de seguir a área futuramente.

Outro fator em questão que merece destaque diz respeito às motivações relatadas para entrada na LA. Ao cruzar os níveis de correlação L-E com as motivações assinaladas, obtivemos quase oito vezes mais chances de escolha correlata no grupo que ingressou na LA por motivos de "Afinidade/Curiosidade" do que no grupo que entrou para aprender algo especifico ou que tinha dificuldade na área (Gráfico 2). Assim, mesmo com a necessidade de complementação curricular discutida por alguns autores como um dos motivos para que a maioria dos estudantes de medicina ingresse em ligas ${ }^{6,7}$, esta motivação, na nossa amostra, não apresentou associação com a escolha de especialidade em área correlata à da liga buscada para este fim. Não sabemos se as expectativas de aprendizado foram frustradas ou mesmo após o fim da sensação de dificuldade na área, o estudante optou por não seguí-la.

O questionário do estudo era composto por questões semi-objetivas, portanto comentários acerca das opções assinaladas não eram possíveis, limitando a análise mais detalhada e profunda dos fenômenos observados. Além disso, a escolha da especialidade médica a ser seguida é complexa e multifatorial, estando presentes desde aspectos mais diretos como afinidade pela área, retorno financeiro, estilo de vida possível para especialidade até a oferta da especialidade na localidade onde reside, tradição familiar (família com médicos que já se consagraram em determinada área), entre outros. Nos diversos trabalhos que avaliam os fatores determinantes para a escolha da 
especialidade médica entre graduandos e médicos que iriam prestar concurso para a residência a afinidade pela área, qualidade de vida e retorno financeiro foram os aspectos mais lembrados ${ }^{8,11,12}$.

Em relação ao papel que as LAs desempenham na escolha da especialização, há poucos estudos sobre os temas. Cavalcante et al. ${ }^{13}$ ao analisarem a produção científica brasileira acerca das Ligas Acadêmicas a fim de identificar lacunas do conhecimento, mostraram que existe precária literatura sobre o tema, trazendo a importância de se refletir sobre o papel das LAs de modo a identificar os aspectos positivos para a graduação ou se servem apenas para uma especialização precoce dos acadêmicos, como sugerem alguns autores analisados.

Assim, apesar do nosso trabalho não sugerir a participação em LA como algo determinante para a escolha da especialidade futura, mais trabalhos são necessários para desígnio do papel que as LAs desempenham na formação médica.

\section{CONCLUSÃO}

Apesar de estar presente no processo formativo da maioria dos estudantes, a participação em Ligas Acadêmicas (LAs) não pareceu representar a “especialização precoce" destes. A percepção de que a participação em LA influenciou a escolha da especialidade foi o fator mais significativamente associado à correlação L-E, isto é, eleger especialidade correlata à Liga Acadêmica participada, sugerindo que esta influência é sentida pelo estudante na direção de favorecimento do contato posterior com a área em questão.

A escolha da especialidade, além disso, é complexa e multidimensional, assim a participação em LAs, quando influente no processo decisório, é apenas um componente da grande rede de fatores imbricados nesta eleição. Per $s i$, isoladamente, esta atividade extracurricular parece não ser determinante para a escolha final da especialidade a ser seguida pelo médico generalista.

AGRADECIMENTOS: Agradeço à Dra. Lara de Araújo Torreão pela aposta realizada no processo de orientação e pelas contribuições ao trabalho. À Comissão Estadual de Residência Médica da Bahia (CEREM/BA) e a Magali, pelo cuidado e suporte durante a matrícula dos residentes, para que a coleta de dados fosse realizada. À Dra. Viviane Sampaio Boaventura de Oliveira, pela revisão plena e cuidadosa de todo o trabalho, assim como pelas valiosas sugestões. A Talita Pontes e Nicolás Rabino pela ajuda na aplicação dos questionários. A Lara Andrade, Bernardo Follador, com carinho, e Ramon Borges pela tradução da versão deste artigo em inglês, aumentando o alcance.

Contribuições dos autores: Sara Pontes: Concepção e desenho da pesquisa; Obtenção de dados; Análise e interpretação dos dados; Análise estatística; Redação do manuscrito. Lara Torreão: Concepção e desenho da pesquisa; Análise e interpretação dos dados; Revisão crítica do manuscrito quanto ao conteúdo intelectual.

\section{REFERÊNCIAS}

1. Pêgo-Fernandes PM, Mariani AW. O ensino médico além da graduação: ligas acadêmicas. Diagn Tratamento. 2011;16(11):50-1. Disponível em: http://files.bvs.br/ upload/S/1413-9979/2011/v16n2/a2048.pdf.

2. Rego S. Currículo paralelo em Medicina, experiência clínica e PBL: uma luz no fim do túnel?. Interface (Botucatu). 1998;2(3):35-48. doi: 10.1590/S1414-32831998000200004.

3. Peres CM, Andrade AdS, Britto SG. Atividades extracurriculares: multiplicidade e diferenciação necessárias ao currículo. Rev Bras Educ Med. 2007;31(3):203-11. doi: 10.1590/S0100-55022007000300002.

4. Ferreira DA, Aranha RN, de Souza MH. Academic leagues: a Brazilian way to teach about cancer in medical universities. BMC Med Educ. 2015;15(236):1-7. doi: 10.1186/s12909015-0524-X.

5. Monteiro LLF, Sacramento Cunha M, Luiz de Oliveira W, Bandeira NG, Menezes JV. Ligas acadêmicas: o que há de positivo? Experiência de implantação da Liga Baiana de Cirurgia Plástica. Rev Bras Cir Plast. 2008;23(3):15861. Disponível em: http://www.rbcp.org.br/details/405/ ligas-academicas--o-que-ha-de-positivo--experiencia-deimplantacao-da-liga-baiana-de-cirurgia-plastica.

6. Lima MC. Ligas Acadêmicas de Medicina da cidade de Belém: caracterização e análise crítica [dissertação]. São Paulo: Universidade Federal de São Paulo; 2013. Disponível em: http://www2.unifesp.br/centros/cedess/producao/teses/ tese_139_ligas_academicas_medicina_mauro_cunha.pdf.

7. Hamamoto Filho PT. Ligas Acadêmicas: motivações e críticas a propósito de um repensar necessário. Rev Bras
Educ Med. 2011;35(4):535-43. doi: 10.1590/S010055022011000400013.

8. De Souza LCL, Mendonça VRR, Garcia GBC, Brandão EC, Barral-Netto M. Medical specialty choice and related factors of brazilian medical students and recent doctors. PloS One. 2015;10(7):e0133585. doi: 10.1371/journal.pone.0133585.

9. Da Silva MdSC, Deslanders A, Sanchez ALdSF, Aníci RAF, Campos LR, Marinho PVS, et al. Fatores e motivações associados à escolha da especialidade Pediatria. Rev Bras Educ Med. 2014;38(4):427-34. doi: 10.1590/S010055022014000400003.

10. Nogueira-Martins MCF, Nogueira-Martins LA, Turato ER. Medical students' perceptions of their learning about the doctor-patient relationship: a qualitative study. Med Edu. 2006;40:322-8. doi: 10.1111/j.1365-2929.2006.02411.

11. Cruz JAS, Sandy NS, Vannucchi TR, Gouveia EM, Passerotti CC, Bruschini H, Srougi M. Fatores determinantes para a escolha da especialidade médica no Brasil. Rev Med (São Paulo). 2010;89(1):32-42. doi: 10.11606/issn.1679-9836.

12. Corsi PR, Fernandes EL, Intelizano PM, Montagnini CCB, Baracat FI, Ribeiro MCSdA. Factors that influence the student's choice of medical specialty. Rev Bras Educ Med. 2014;38(2):213-20. doi: 10.1590/S010055022014000200008 .

13. Cavalcante ASP, Vasconcelos MIO, Lira GV, Henriques RLM, Albuquerque INM, Maciel GP, Ribeiro MA, Gomes DF. The Academic Leagues in the Health Area: Knowledge Gaps from the Brazilian Scientific Production. Rev Bras Educ Med. 2018;42(1):199-206. doi: 10.1590/1981-52712018v42n1rb20170081.

Recebido: 22.03.19 Aceito: 26.06.19 